\title{
Photochemical Decomposition of Pesticide Chemicals in Aerosol Particles and Thin Films Relevant to Environmental Conditions
}

\author{
Yuri N. Samsonov* \\ Siberian Branch of the Russian Academy of Sciences, Institute of Chemical Kinetics and Combustion, Novosibirsk 630090, Russia
}

Received: November 24, 2013 / Accepted: December 10, 2013 / Published: March 25, 2014.

\begin{abstract}
Contamination of the environment by pesticides is the inevitable aftermath of plant protection, and a substantial portion of pesticide pollutants exists in the form of aerosol particles levitated in the air and deposited on plants, and as the pesticide residues (thin films) on the surface of plant leaves. The sunlight photolysis could be the resource for the accelerated photochemical decomposition of pesticide compounds to minimize the long-term environmental contamination. The rates of photochemical decomposition of pesticide chemicals propiconazole (commercial formulation Tilt) and haloxyfop-ethoxyethyl (Zellek) were measured in particles of $0.12-1.3 \mu \mathrm{m}$ in diameter and in films $0.04-0.2 \mu \mathrm{m}$ thick. A specific polyaromatic sensitizer Shirvanol was used to induce accelerated decomposition of the above pesticide particulates under both the solar radiation and the artificial UV light. It was established that propiconazole decomposes by the sensitized photo-oxidation only, but haloxyfop-ethoxyethyl reacts in both the oxygen (air) and oxygen-free mediums via both the direct and sensitized reactions. The photochemical mechanisms are hypothesized and argued for the oxidative and non-oxidative decompositions. The haloxyfop-ethoxyethyl (Zellek) residues formed on foliage upon pesticide treatments of agricultural fields would essentially decompose under sunlight via a direct photoreaction in 4-6 weeks, but the propiconazole (Tilt) contaminants probably need more time.
\end{abstract}

Key words: Pesticide, propiconazole, haloxyfop-ethoxyethyl, aerosol particles, thin films, photochemical decomposition.

\section{Introduction}

The disperse state of chemical substances is a natural phenomenon. Hundreds millions of tons of particulate matter enter the atmosphere annually from both the natural and anthropogenic sources. Anthropogenic contribution is caused by heat-and-power engineering, motor fuel combustion, and application of pesticide chemicals in agriculture. Reactions of pesticide compounds, which permanently proceed in the environment (reactions with $\mathrm{OH}$ radicals, nitrogen oxides and ozone, (photo-) chemical and (photo-) catalytic reactions, etc.) result in decomposition of pesticide molecules. Decomposition

\footnotetext{
"Corresponding author: Yuri N. Samsonov, Ph.D., research fields: application of pesticide aerosols in agriculture and forestry, photochemistry of pesticide contaminants in environment. E-mail: samsonov@kinetics.nsc.ru.
}

induced by sunlight seems to be very important for the fate of pesticide pollutants in the environment. The photochemical stage of decomposition, which generally is the many-stage physicochemical process, often predetermines the overall decomposition rate.

Many articles, books and reviews report the reactions between the chemical contaminants and the active reagents in the air [1-11]. Since the reagents $\left(\mathrm{OH}, \mathrm{O}_{3}, \mathrm{NO}\right.$ and others) arise, as a rule, by the primary action of the sunlight, the term "photochemical reaction" is conditionally applied to all chemical reactions in which these reagents participate. Several papers report the reactions between $\mathrm{OH}$ radicals (ozone, nitric oxides) and organic compounds in the form of layers of chemical substances on glass plates placed in a tube [5, 6, 12, 13]. The air containing the active reagents under study 
flows through the tube. These flow-tube experiments simulate the (photo-) chemical reactions with residues of both the pesticide and the non-pesticide compounds deposited from the air onto plant foliage. Photochemical decomposition of several pesticide compounds was studied in the form of either the liquid solutions in glass test-tubes or the (semi-) solid films formed on glass plates [14-19]. The reaction rates and the quantum yields were measured under action of both the solar radiation and the laboratory sources of actinic light. The decomposition products were partly recognized, and those were used to speculate on the pathways of chemical conversions of the pesticide ingredients and the other compounds, which compose the pesticide formulations. However, the kinetic dependences were not measured under different conditions. Besides, the preceding and concurrent physicochemical transformations were not taken into account, which permanently proceed in the disperse pesticide matter in the environment. The pesticide films under study as well as the pesticide layers used in the above flow-tube experiments were rather coarse entities 20-30 $\mu \mathrm{m}$ thick and more. As it is explained in Section 1.1, there are specific features of physicochemical conversions and photochemical reactions which proceed in the high disperse substances, i.e., in fine aerosol particles and very thin films.

\subsection{Specificity of Photochemical Reactions in High Disperse Matter}

The light absorbed in bodies of fine particles and thin films can induce chemical reactions inside. There is the lack of reference data on the intra-particle absorption of light preceded or/and followed by the physicochemical transformations and chemical changes. The photochemical destruction of polymeric films partly concerns these problems [20-22]. However, the polymeric films are generally 20-100 $\mu \mathrm{m}$ thick, their chemical compositions are known, and the bulk structures are fixed. As it will be explained below, both the compositions and the structures of pesticide particles and films are changeable and often irreproducible, especially under ambient conditions. As for the photochemical reactions in the high disperse substances, it is worth noting that the photochemical data obtained by irradiating pesticide compounds in the ordinary "test-tube" solutions cannot, as a rule, be adequate to the physicochemical and photochemical processes which proceed in fine particles and films. Moreover, the same holds for the coarse particles and films several tens and hundreds of micrometers thick, because the high disperse substance state manifests specificities for rates and mechnisms of physicochemical transformations and photochemical reactions inside fine particles and films. Some of them are as follows:

(1) The rates of vaporization of chemical compounds out of particles (or film) are known to be proportional to the saturation pressures of compounds and the inverse squared particle's diameter (inverse film's thickness). Consequently, during vaporization of volatile compounds (e.g., solvents) from a system of poly-disperse and chemically multi-component particles, the chemical composition and size of a particle are permanently changing. The quickest changes progress in the smallest particles, so that the chemical compositions of the particles (films) of different sizes should be variable, and dissimilar from their initial composition (it was the same in all particles just after dispersion), and from each other.

(2) In the course of solvent vaporization, a particle (film) can gradually change its phase condition from the initial liquid formulation to a solid matter (better to say, to a semi-solid matter, i.e., porous, friable, viscous). The solid and viscous conditions diminish the diffusivity of reacting molecules (e.g., oxygen) by several orders of magnitude as compared with the liquid state. On the other hand, a feasible porous structure of hardened particles promotes penetration of oxygen and other air reagents $\left(\mathrm{OH}, \mathrm{NO}, \mathrm{O}_{3}\right)$ directly into the particle's body. The uncertain dependence of 
the diffusion rates on the phase condition and the bulk structure complicates the diffusion-controlled chemical reactions in the bodies of particles and films.

(3) The solidification of the intra-particle matter mentioned above can cause the partial and even full stratification of the remaining compounds. In the initial particle, all the compounds were dissolved in a liquid solvent (the compounds are assumed solid). After solvent(s) vaporization, the hardened compounds can be mutually insoluble. Thermodynamically, each insoluble compound should form the separate volume portion in the particle's body. In many cases, however, such separation is inaccessible as it should be accompanied by formation of interfacial surfaces isolating the volume portions from each other. This requires some energy that prevents the stratification of the intra-particle matter. Consequently, these components can long live in the interior of particles and thin films as a homogeneous solid solution. This non-equilibrium solution is called the "meta-stable solution", and it is known in physical chemistry even in large samples. It is evident, however, that formation of meta-stable states is most likely in fine particles and films. Actually, the surface energy which is required for the stratification delay is proportional to the surface size (i.e., squared particle diameter), but the energy that is favorable for volume stratification is proportional to the volume (cubed diameter). Thus, the "surface energy/volume energy" ratio is greater for fine particles, and, therefore, the meta-stable states should arise in the high disperse systems. It may be then supposed that many chemical reactions in a hardened matter proceed owing to the long-living meta-stable conditions. If the meta-stable matter spontaneously transforms to the equilibrium, the reaction can terminate because of reagent separation.

Thus, the mechanisms and rates of chemical reactions in fine particles and thin films differ from the reactions in ordinary liquid solutions, as well as in the coarse particles and films. The point is that, taking into account the inverse squared diameter dependence of vaporization rate on particle sizes, one may perceive that the solvent vaporization out of the coarse particles and the thick films, and, consequently, solidification-and-stratification of intra-particle matter require many hours and days (certain kinds of solvents used in commercial pesticide formulations are of low and middle volatilities) as compared with a few minutes in the sub-micron particles and films.

\subsection{Photodecomposition of Pesticide Pollutants Relevant to Ambient Conditions}

One of the characteristic properties of high disperse substances is that only the low-volatile organic (pesticide) compounds with molecular weights of 300-500 g. $\mathrm{mol}^{-1}$ and more, and with saturation pressure of $10^{-4}-10^{-8} \mathrm{~Pa}$ and lower can form fine films and particles (e.g., $1 \mu \mathrm{m}$ in diameter), which could live in the environment for many hours, days and weeks [23]. The particles formed from the "light" compounds of $100-200 \mathrm{~g} \cdot \mathrm{mol}^{-1}\left(10^{-2} \mathrm{~Pa}\right.$ and higher) should vaporize very quickly (few hours, minutes and seconds). One can calculate that the mass of a one-micron particle is less than $10^{-12} \mathrm{~g}$. It is practically impossible to detect chemical changes in such a tiny particle. It is necessary to experiment with a group of at least $10^{6}$ particles. The weight of this particulate system (or a thin film of $0.1 \mu \mathrm{m}$ and less in thickness) is within a few fractions of microgram to several micrograms, but such small mass requires the specific kinds of chemicals and the sensitive methods of chemical analysis. Most of pesticide chemicals are very low-volatile compounds, which can live long in the environment in the form of fine aerosols and thin films. Many kinds of pesticides (e.g., chlorine-containing) can be detected by gas chromatography at very low concentrations using an electron capture detector to register changes in chemical composition of the particulate systems mentioned above.

The annual amount of pesticide chemicals used in 
the world agriculture is estimated at 1-2 million tons. Various engineering techniques are known to disperse the commercial pesticide formulations over agricultural fields - the special engines which disperse liquid pesticide formulations into small aerosol particles; the aerial, blower, and boom sprayers which produce the "pesticide formulation-and-water" droplets. Both the particles and droplets fall onto plant leaves forming here either a layer of deposited particles or a thin pesticide film (residue, spot) 0.01-1 $\mu \mathrm{m}$ thick. As for the pesticide films on leaf's surface, they are not the perfect films but rather the fragmentary (discontinuous) entities composed of the spread pesticide matter or the clustered emulsion particles. Therefore, the 0.01-1 $\mu \mathrm{m}$ film thickness mentioned above should be considered as the conditional measure estimated from a quantity of pesticide matter in a droplet spread over the leaf surface. Structurally, these films can be in amorphous, semi-liquid (viscous), semi-solid (porous) and, unlikely, crystalline states. The shapes, and the chemical and bulk conditions of particles, droplets and films are diverse depending on the chemical and phase properties of the pesticide and non-pesticide compounds which compose the commercial pesticide formulations.

Pesticides are reputed to be the ecologically dangerous air and vegetation contaminants. Negative ecological consequences of pesticide application are often assigned to their persistence in the environment. Ideally, pesticide residues should persist on foliage for the period required for pest control (a few hours or days) but then they should decompose to minimize the environmental contamination. The sunlight photolysis could be the resource for chemical decomposition in both the levitated pesticide aerosols, in the pesticide particles deposited on foliage, and in the pesticide films (residues, spots) spread over leaf's surface. However, most of pesticide compounds have a spectral absorption shorter than $300 \mathrm{~nm}$, whereas the solar radiation shorter $300 \mathrm{~nm}$ is absorbed by stratospheric ozone and, hence, fails to reach the land. Therefore, most of pesticide pollutants manifest the photochemical stability in the environment. A possible means of regulating decomposition rates can be addition of a special sensitizer into a pesticide formulation to absorb the near-land sunlight followed by transfer of this energy to the pesticide molecules, which then should decompose. Several regulating chemicals have been experimented in 70-80 s, but, for some reasons, these sensitizers have found no practical use in plant protection [14, 15, 17, 18, 24]. The authors have earlier studied the photochemical interaction between several pesticide chemicals and sensitizers in the form of fine particles and thin films in the outdoor and laboratory conditions [25-27]. When varying the concentration of sensitizer, the pesticide lifetimes were regulated from $2-3 \mathrm{~h}$ to several days.

The objective of this study was to measure the rates, and to hypothesize the mechanisms of both the direct and sensitized photodecompositions of two pesticide chemicals in the form of fine particles and thin films relevant to the environmental conditions, with the specificities of high disperse pesticide particulates being taken into account (as it is stated above, the pesticide residues in agricultural plants are, for the most part, the thin films less that $1 \mu \mathrm{m}$ thick). The outdoor photochemical experiments were conducted under solar radiation in summer seasons of 2008-2010, near Novosibirsk, Russia (55N, 83E). The indoor experiments were carried out in Laboratory of Dispersal Systems, the Institute of Chemical Kinetics and Combustion, Novosibirsk.

\section{Materials and Methods}

\subsection{Pesticide Chemicals and Analysis Technique}

The pesticide films and particles made of either the commercial pesticide formulation Tilt (emulsion concentrate, Ciba Geigy-Novartis) or its active ingredient "propiconazole" (generic name) were experimented in this study. This formulation with 
fungicidal agent propiconazole is widely applied at wheat fields. The formulation, except $250 \mathrm{~g} \cdot \mathrm{L}^{-1}$ of propiconazole, contains a solvent and the minor fractions of emulsifier and stabilizer. A small assay of pure propiconazole was prepared from Tilt by solvent distillation under vacuum $\left(\approx 200{ }^{\circ} \mathrm{C}, \approx 70 \mathrm{~Pa}\right)$ followed by water washing to remove emulsifier, drying, and purification in a column packed with $\mathrm{Al}_{2} \mathrm{O}_{3}$. The $97 \%-98 \%$ purity of propiconazole was checked by gas-liquid chromatography with a flame-ionization detector. From a reference book, it is a tintless viscous liquid, but a $2 \%-3 \%$ resinification occurring upon the high-temperature distillation made the assay yellowy. The chemical formula of propiconazole is $\mathrm{C}_{15} \mathrm{H}_{17} \mathrm{Cl}_{2} \mathrm{~N}_{3} \mathrm{O}_{2}$, the molecular weight is $342 \mathrm{~g} \cdot \mathrm{mol}^{-1}$, the molecular structure is shown in Scheme 1.

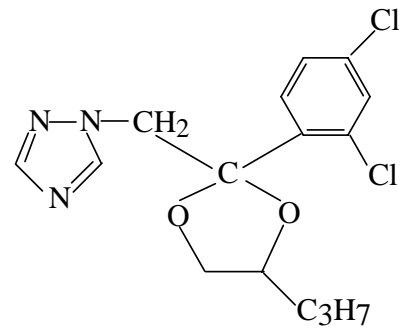

Scheme 1 The structural formula of propiconazole.

The emulsion concentrate Zellek (Dow Agro, Zellek is used as a herbicide substance for protection of various technical crops) contains $125 \mathrm{~g} \cdot \mathrm{L}^{-1}$ of haloxyfop-ethoxyethyl (generic name), $\mathrm{C}_{19} \mathrm{H}_{19} \mathrm{ClF}_{3} \mathrm{NO}_{5}$ (the structural formula is shown in Scheme 2), $434 \mathrm{~g} \cdot \mathrm{mol}^{-1}$. This formulation was used to study physicochemical and photochemical conversions in thin films.

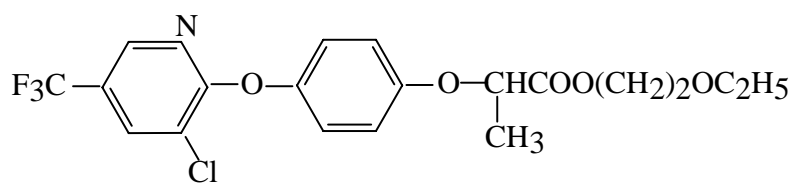

Scheme 2 The structural formula of $\mathrm{C}_{19} \mathrm{H}_{19} \mathrm{ClF}_{3} \mathrm{NO}_{5}$.

A Shirvanol 2 sensitizer was used to regulate photolysis rates. It is a by-product of the catalytic cracking of Caspian oil [28]. It consists of naphthenic hydrocarbons ( $60 \%$ by weight), and $40 \%$ of polycyclic aromatics such as naphtalene, phenanthrene, and anthracene. These aromatics are well known as photo-sensitizers, and they were used for the induced photo-degradation (aging) of polymers [21, 22], and for the regulating decomposition of pesticide residues $[15,24]$. The liquid naphthenic hydrocarbons serve here as a composite solvent that is suitable for dissolving the pesticide chemicals under study.

A gas-liquid chromatograph was used to detect the quantities of propiconazole and haloxyfop-ethoxyethyl in fine particles and films, and the products of photochemical reactions. A glass column of $3 \mathrm{~mm}$ ID and $50 \mathrm{~cm}$ long was packed with a 5\% SE-30 on a Chromaton-N-AW-DMCS (0.16-0.2 mm), the oven temperature was $230{ }^{\circ} \mathrm{C}$. A very sensitive electron-capture detector enables us to experiment with a film 0.01-0.5 $\mu \mathrm{m}$ thick, and a system of sub-micron aerosols deposited on glass plate (a pesticide quantity in this particles and films was within $0.3-20 \mu \mathrm{g})$. A HP $6890 \mathrm{~N} / 5973 \mathrm{~N}$ gas chromatograph-mass spectrometer was used in attempt to recognize the decomposition products.

\subsection{Preparation of Pesticide Films and Aerosol Particles and Photolytic Procedure}

Several acetone solutions, containing the given concentrations of pure propiconazole or the Tilt formulation $\left(100-5,000 \mu \mathrm{g} \cdot \mathrm{mL}^{-1}\right.$ of active ingredient) and Shirvanol $\left(100-5,000 \mu \mathrm{g} \cdot \mathrm{mL}^{-1}\right)$, were prepared beforehand with the different "sensitizer/propiconazole" ratios, $z$. The ratio varied from $z=0$ (absence of sensitizer) to $z \approx 6$ in different solutions. The "sensitizer/haloxyfop-ethoxyethyl" mixtures were prepared in similar manner.

Glass plates of $3.5 \times 2.5 \mathrm{~cm}$ were used to form thin pesticide films. The front sides of plates were polished with a fine abrasive to create a matted surface. Solution with a given $z$ and a given pesticide concentration was spread over the plate with a pipette (0.03-0.08 $\mathrm{mL})$ to provide the given surface concentrations of sensitizer, $0-30 \mu \mathrm{g} \cdot \mathrm{cm}^{-2}$, and pesticide, 1-10 $\mu \mathrm{g} \cdot \mathrm{cm}^{-2}$. After evaporation of acetone 
(and volatile solvents of the Shirvanol, Tilt, and Zellek formulations too), a thin film composed of the given "sensitizer-and-pesticide" mixture of 3-40 $\mu \mathrm{g} \cdot \mathrm{cm}^{-2}$ in total surface concentration forms on the plate. Nominally, the above surface concentrations correspond to films of $0.03-0.4 \mu \mathrm{m}$ in the rated thickness (for reference, the $100 \mu \mathrm{g} \cdot \mathrm{cm}^{-2}$ concentration corresponds to the $1 \mu \mathrm{m}$ thickness, assuming the specific density of "sensitizer-and-pesticide" matter of $\left.1 \mathrm{~g} \cdot \mathrm{cm}^{-3}\right)$. Visually, the films look as the smooth (sometimes, slick) matter especially the "thick" films of 0.2-0.5 $\mu \mathrm{m}$, and just the matted surface is suitable for forming smooth films (the films formed on a non-polished glass sometimes tend to partial coagulation).

In outdoor experiments, 8-10 plates were exposed to solar radiation. With a periodicity of 3-6 h two plates were taken away. The plates were jet-washed with a hexane: acetone solvent (5:1 by volume, $3-5 \mathrm{~mL}$ ). The extracts were analyzed chromatographically to determine the pesticide quantities retained in the films upon exposure (the total exposure was sometimes 15 and more hours). Several plates were kept in the dark to refer to the initial pesticide quantities (owing to low volatility of pesticide ingredients no evaporation loss was recorded in the dark).

A similar procedure was used in the indoor experiments with the artificial (lamp) light. Several plates were placed at the flat bottom of metallic cuvette at distance of 12-15 cm from the lamp. The cuvette was cooled with cold water. Additionally, it was covered with a glass plate to cut off infrared radiation from the lamp and, thus, to prevent the films from heating. The glass served also as an UV-filter to cut off the light shorter than $300 \mathrm{~nm}$ (the "White Glass" filters WG3 and WG4 were used). The cuvette consists of two leak-free sections which can be filled with different gases. This allows us to study photochemical reactions in different gas media, e.g., with and without oxygen.

Pesticide aerosols were produced from the Tilt formulation using the DeVilbiss pneumatic sprayer [29]. The size distribution of particles was measured with an optical particle-size analyzer, and it was found to be the lognormal one with the mass-median diameter $d_{m} \approx$ $2 \mu \mathrm{m}$, and the geometrical standard deviation $\sigma_{g} \approx 1.8$. This means that $95 \%$ of the mass were in the particles ranging from $0.6 \mu \mathrm{m}\left(d_{m} \cdot \sigma_{g}^{-2}\right)$ to $6.5 \mu \mathrm{m}\left(d_{m} \cdot \sigma_{g}^{2}\right)$. As the particles suitable for our experiments should be sometimes smaller than the originally produced ones, Tilt was pre-diluted with xylene (1 to 125 by volume). The xylene evaporation caused a 5-fold size reduction. Thus, $95 \%$ of aerosols ranged within $\approx 0.12-1.3 \mu \mathrm{m}$ in these experiments. The "air-and-aerosols" mixture from the sprayer flowed in a $190 \mathrm{~L}$ chamber. The pesticide aerosols deposited onto the $2.7 \times 7.5 \mathrm{~cm}$ glass plates placed at the bottom beforehand forming a "monolayer" of particles $0.12-1.3 \mu \mathrm{m}$ thick. The plates with particles were exposed to a long-term irradiation simulating the slow sunlight photolysis of pesticide particles deposited on plants. It was impossible to experiment with the levitated particles due to the permanent deposition of particles onto chamber's walls and bottom, which results in the uncontrolled decrease of concentrations. Although the deposited aerosols were not the perfect simulation of particles freely levitated in the air, they reasonably good simulated physicochemical conversions and photochemical reactions in the environment. As for the films on plates (they simulated the pesticide residues on foliage), they should be considered as the high disperse matter too in spite of the fact that they are of $2.5 \times 3.5 \mathrm{~cm}$ in surface sizes. The point is that just the thickness, $0.03-0.5 \mu \mathrm{m}$ in our case, but not the transversal sizes characterizes the high disperse properties.

\subsection{Absorption Spectrums of Pesticide Chemicals and Laboratory Source of Actinic UV Light}

Absorption spectrums of pesticide chemicals were recorded using a Specord M40 spectrometer (Carl Zeiss, Germany). The spectrums were recorded in either a solution (using iso-propanol and hexane as 
solvents) or a (semi-) solid film formed after solvent(s) vaporization out of a solution layer spread over a quartz plate (Section 2.2). Fig. 1 shows the long-wave sides, 260-400 nm, of the UV absorption spectrums of pesticides propiconazole and acrinathrin (the acrinathrin spectrum is used in Section 3.2), which border with the UV edge of the near-land solar radiation. Although a noticeable absorption at $\lambda>300$ $\mathrm{nm}$ (it is a conventional short-wave edge of the near-land sunlight) is observed for the propiconazole assay but it was found that this absorption is caused not by the propiconazole molecules but by the resinous impurities mentioned in Section 2.1 (additional evidence is in Section 3.1). The Tilt formulation manifests a significant absorption at $\lambda>$ $300 \mathrm{~nm}$ too, but the non-pesticide ingredients of formulation (solvent, emulsifier, etc.) cause this absorption (Section 3.1). A spectrum of the Zellek formulation is shown in Fig. 1 too, but there is no haloxyfop-ethoxyethyl spectrum for the absence of the purified ingredient. As for the Shirvanol 2 sensitizer, it strongly absorbs the actinic solar radiation within 300-380 nm. It should be noted that for demonstration of the absorption spectrums, rather high concentrations were used in Fig. 1, which correspond to the equivalent film thickness approximately of 5 $\mu \mathrm{m}$, as no appreciable absorption would be recorded in the $0.1 \mu \mathrm{m}$ films under study.

Fig. 1 shows also the actinic solar radiation near the land (Novosibirsk, $55^{\circ} \mathrm{N}, 83^{\circ} \mathrm{E}$, Russia). It was calculated assuming a clear-sky weather at summer solstice, at noontime (solar zenith angle is about $32^{\circ}$ ) and at morning and evening $\left(80^{\circ}\right)$, taking into account the reference data on spectral irradiance above the stratosphere, the absorption of the UV light by a typical stratospheric ozone layer of $\approx 340-350$ DU (Dobson Units) over Siberian territory, as well as the Rayleigh scattering and the light attenuation by the so-called "particulate diffusion of light" (scattering + reflection + refraction + diffraction by atmospheric particulates) in the troposphere. One can see that the

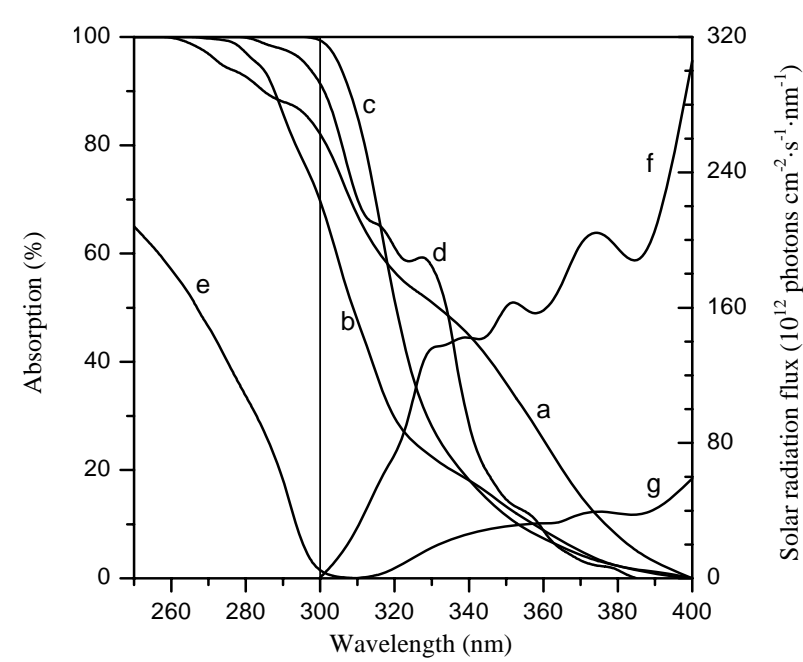

Fig. 1 The long-wave sides of absorption spectrums of pesticide chemicals (left Y-axis): (a)-solution of propiconazole in isopropanol, $500 \mu \mathrm{g} \cdot \mathrm{mL}^{-1}$, cell thickness 1 cm; (b)-Tilt in isopropanol, $500 \mu \mathrm{g} \cdot \mathrm{mL}^{-1}$ of active ingredient propiconazole; (c)-Zellek in isopropanol, 500 $\mu \mathrm{g} \cdot \mathrm{mL}^{-1}$ of active ingredient haloxyfop-ethoxyethyl; (d)-Shirvanol 2, $30 \mu \mathrm{g} \cdot \mathrm{mL}^{-1}$ in hexane; (e)—acrinathrin as a solid film on quartz plate, surface concentration 70 $\mu \mathrm{g} \cdot \mathrm{cm}^{-2}$. The near-land solar irradiance (right Y-axis): (f)-summer solstice, noontime (zenith angle $32^{\circ}$ ); (g) - morning and evening $\left(8^{\circ}\right)$. Vertical line at $\lambda=300$ nm-the conventional short-wave edge of near-land sunlight.

UV light shorter $300 \mathrm{~nm}$ is practically absent in the near-land irradiance and, consequently, there should be (almost) no absorption of the actinic 300-400 nm light by the pesticides under study.

In laboratory conditions, a quartz tungsten halogen lamp was used to simulate solar radiation. Generally, its spectrum differs from the near-land sunlight. On the other hand, we need to simulate only a spectral range of $300-400 \mathrm{~nm}$ that corresponds to both the near-land solar UV radiation and the absorption by Shirvanol. Placing a spectral filter (WG3, WG4) between the lamp and the plates with the films, and varying the distance between them, we can mimic the photochemical action of sunlight. It is worth noting that the perfect spectral simulation of solar radiation is not the essential condition in such experiments but its resultant photochemical action is only required. The point is that the particular efficiency of photochemical reaction (the so-called quantum yield) is, as a rule, independent on the wavelength within a certain 
spectral range. Therefore, a smoothed irradiance distribution within the $300-400 \mathrm{~nm}$ range would be applicative resulting in close decomposition rates under both the solar radiation and the laboratory light.

\section{Results and Discussion}

3.1 Photodecomposition Rates of Propiconazole (Tilt) and Haloxyfop-Ethoxyethyl (Zellek) in High Disperse Particulates

As indicated earlier [25-27], the reaction kinetics often presents the first-order rate law $Q(t)=Q_{0}$ $\exp \left(-k_{\text {eff }} t\right)$, where $t$ is the exposure time $(h), Q_{0}$ and $Q(t)$ are, respectively, the initial and current amounts of pesticide ingredient; $k_{\text {eff }}$ is the efficient rate constant $\left(\mathrm{h}^{-1}\right)$. Actually, photochemical decomposition is a multi-stage process including light absorption, excitation energy transfer and quenching, physicochemical conversions and chemical reactions. Thus, the first-order equation is the pseudo-kinetic law, which depends on the physical and chemical processes listed above (the pseudo first-order dependences of photochemical reactions are well known $[8,30]$ ).

Fig. 2 shows the first-order kinetics for long-term exposure, $16-28 \mathrm{~h}$, of films $\approx 0.07-0.1 \mu \mathrm{m}$ thick made of the purified propiconazole, Tilt (propiconazole), and Zellek (haloxyfop-ethoxyethyl). The sensitizing agent Shirvanol was added at $z \approx 1$. Photolysis was conducted in both the outdoor and indoor conditions. As is evident from Fig. 2, the sensitized conversion follows the first-order law if a conversion depth is less than $40 \%-50 \%$, but then the rates progressively lower (therefore, the fitted lines are not shown to the $50 \%-70 \%$ conversion depth). This is most likely caused by gradual change of the phase and bulk conditions in the irradiated films. This hinders the intra-film diffusivity of reagents, e.g., oxygen (the gradual stagnation was also observed in pesticide formulation Adonis (fipronil) [27]).

The decomposition rates were rather close in the propiconazole films irradiated by either the solar or indoor radiation (more correctly, the indoor radiation

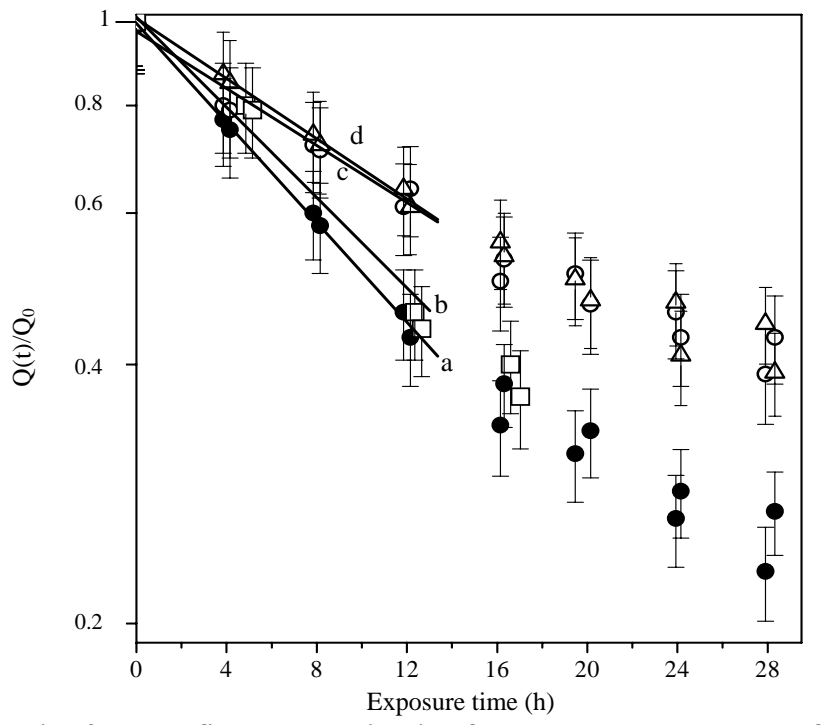

Fig. 2 The first-order kinetics for long-term exposure of films $\quad 0.07-0.1 \quad \mu \mathrm{m} \quad$ thick made of propiconazole-and-sensitizer composition, lamp radiation, $z$ $\approx 1$ (symbols $\quad \bullet, \quad$ straight line a); propiconazole-and-sensitizer, solar radiation, $z \approx 1$ (symbols, line b); Tilt-and-sensitizer, lamp radiation, $z \approx 1$ (o, line c); Zellek-and-sensitizer, lamp radiation, $z \approx 1(\Delta$, line d).

was adjusted to provide such a similarity, Section 2.3). On the other hand, as the solar radiation regularly changed during the day the outdoor rates changed too (the noontime rate in the 5-12 $\mathrm{h}$ time period was somewhat higher than in the morning and evening, the 0-5 and 12-16 h periods).

Fig. 3 shows the rate constant $k_{\text {eff }}$ as a function of the "sensitizer/propiconazole" ratio $z$ in films $\approx$ 0.05-0.07 $\mu \mathrm{m}$ thick. The experiments were conducted under the lamp radiation that simulated the actinic sunlight, but the close dependence was obtained under solar radiation too (but it is not here shown in Fig. 3). The films were prepared from the "sensitizer-andpropiconazole" and the "sensitizer-and-Tilt" mixtures. The data in Fig. 3 are nominally described by the fitted curves $k_{\text {eff }}=0.098 \mathrm{z} /(\mathrm{z}+1.52)$ for "sensitizer-and-Tilt", and $k_{\text {eff }}=0.13 z /(z+1.01)$ for films made from "sensitizer -and-propiconazole". From these formulas, one can estimate a sensitizer amount to add in Tilt to achieve a given decomposition rate in the environment (this conclusion is only hypothetical but not practical 


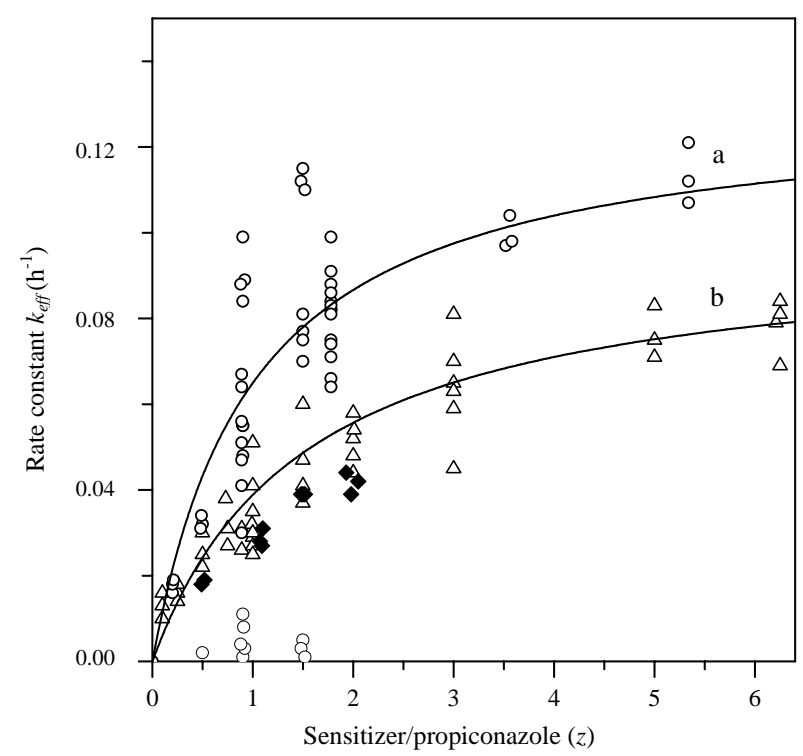

Fig. 3 The decomposition rate constants $k_{\text {eff }}$ of propiconazole and Tilt as a function of the "sensitizer/propiconazole" ratio $z$ : symbols o and a fitted (a) - films $\approx 0.05-0.07 \mu \mathrm{m}$ thick $\left(5-7 \mu \mathrm{g} \cdot \mathrm{cm}^{-2}\right)$ made from the propiconazole-and-sensitizer composition; $\Delta$ and (b)—films made from the Tilt-and-sensitizer composition; $\bullet$-particle of $\approx 0.12-1.3 \mu \mathrm{m}$ in diameter made from a Tilt-and-sensitizer mixture.

as an additional medicobiological research should be needed). Decomposition rates in the films made of "sensitizer-and-Tilt" are considerably lower than in the "sensitizer-and-propiconazole" films, whereas for several earlier studied pesticide formulations these rates were close to each other [26]. This is not surprising as the current chemical and structural conditions in both kinds of films could differ from each other. Most likely, a solvent used in Tilt is a middle volatile compound (probably, homolog(s) of tetraline) that persisted in films for several hours and, thus, acted as buffer or/and excitation quencher (more volatile solvent was in other pesticide formulations, e.g., xylene in Rufast (acrinathrin) [26]).

The data points in Fig. 3 demonstrate an extremely wide spread of rates but this is not the trivial measurement inaccuracy (the pooled uncertainties including the chromatographic ones were within 10\%-15\%). This phenomenon we observed before [26, 27], but no other papers are known, which have reported such an effect. Most likely, the wide spread is caused by irregular hardening and partial stratification of film's matter, wherein the sensitizer and pesticide substances could separate from each other (this effect is explained in Section 1.1). Physically, the stratification of intra-film matter could arise only in a small portion of film's volume but, sometimes, stratification could spread over the entire volume. Some rate points in Fig. 3 show very low values, so they should be referred to the almost fully stratified films. This occurs mostly at $z \approx 1-1.5$, corresponding to (approximately) a 1:1 "sensitizer:propiconazole" mixture, where poor mutual solubility of both ingredients can promote separation of reagents. This effect was weaker in the "sensitizer-and-Tilt" films, most likely due to the retarding vaporization of Tilt's solvent resulting in stratification delay. The maximal rate points refer probably to the long-living homogeneous "sensitizer-and-propiconazole" mixture in the films (a meta-stable solution, Section 1.1). As it is explained in Section 1.1, this is a specific feature of sensitized photodecomposition in a high disperse pesticide matter.

Several experiments were conducted using aerosol particles deposited on glass plates (the 0.12-1.3 $\mu \mathrm{m}$ particles were made of the “xelene-and-Tilt-and-sensitizer" solution followed by evaporation of xelene, Section 2.2). As seen in Fig. 3, there are no dramatic differences of photolysis rates in the $0.05-0.2 \mu \mathrm{m}$ films (the rate constants for different films changed not faster than $1 / \sqrt{h}$, where $h$ is the film thickness) and the $0.12-1.3 \mu \mathrm{m}$ aerosols settled on the plates. The systematically lower rates in aerosols were due to the fact that the actinic UV light can be absorbed almost completely by the outer layer, $\approx 0.5-1$ $\mu \mathrm{m}$ thick, of particle's volume (poly-aromatic compounds of Shirvanol have such a strong absorption, $\approx 19,000 \mathrm{~cm}^{2} \cdot \mathrm{g}^{-1}$ at $\lambda=313 \mathrm{~nm}, \approx 3400$ $\mathrm{cm}^{2} \cdot \mathrm{g}^{-1}$ at $\lambda=365 \mathrm{~nm}$, our data). Therefore, the inner volume in the $0.5-1.3 \mu \mathrm{m}$ fraction of particles was inaccessible for the light, which resulted in lessened rates. Although the deposited aerosols were not the 
perfect simulation of particles levitated in the air, they were suitable for study the physicochemical and photochemical conversions of pesticide particles in the environment, both deposited on plants and dissipated in the atmosphere.

As seen in Fig. 3, there is no direct photodecomposition of propiconazole (Tilt) at $z=0$ (the efficient rate was less than $0.002 \mathrm{~h}^{-1}$ ). This confirms the assumption that the light absorption at $\lambda>300 \mathrm{~nm}$ is caused by the resinous impurities in the purified propiconazole and the non-pesticide components of Tilt rather than by propiconazole molecules themselves. Conducting photolysis in either oxygen (air) or oxygen-free (argon) medium indicates the absence of an oxygen-free reaction in films made from the propiconazole-and-sensitizer mixtures. In this regard, the sensitized decomposition of propiconazole is actually the photochemical oxidation induced by sensitizer (a mechanism of the sensitized photo-oxidation is proposed in Section 3.2).

Fig. 4 shows the efficient rate constants as a function of the "sensitizer/haloxyfop-ethoxyethyl" ratio in films $\approx 0.07 \mu \mathrm{m}$ thick (the thicknesses ranged within 0.04-0.2 $\mu \mathrm{m}$ in different sets of experiments). The films were prepared from the Zellek formulation mixed with the Shirvanol sensitizer. Generally, the curve shape in Fig. $4, k_{\text {eff }} \approx 0.01+0.045 z /(z+0.3)$ is similar to the propiconazole and Tilt curves in Fig. 3. However, haloxyfop-ethoxyethyl demonstrates a significant direct photodecomposition $k_{\text {eff }} \approx$ 0.007-0.012 $\mathrm{h}^{-1}$ at $\mathrm{z}=0$ (this fact indicates that haloxyfop-ethoxyethyl absorbs the sunlight at $\lambda>300$ $\mathrm{nm})$. Second, it decomposes both with and without oxygen (the oxygen-free rate, $k_{\text {eff }} \approx 0.006+0.027$ $z /(z+1.6)$, is $2-3$ times lower than the oxygen one). Thus, photodecomposition of haloxyfopethoxyethyl in thin films (most probably, in aerosol particles too) proceeds concurrently by the direct and induced reactions, and by the oxidative and non-oxidative pathways (possible mechanisms are proposed below).

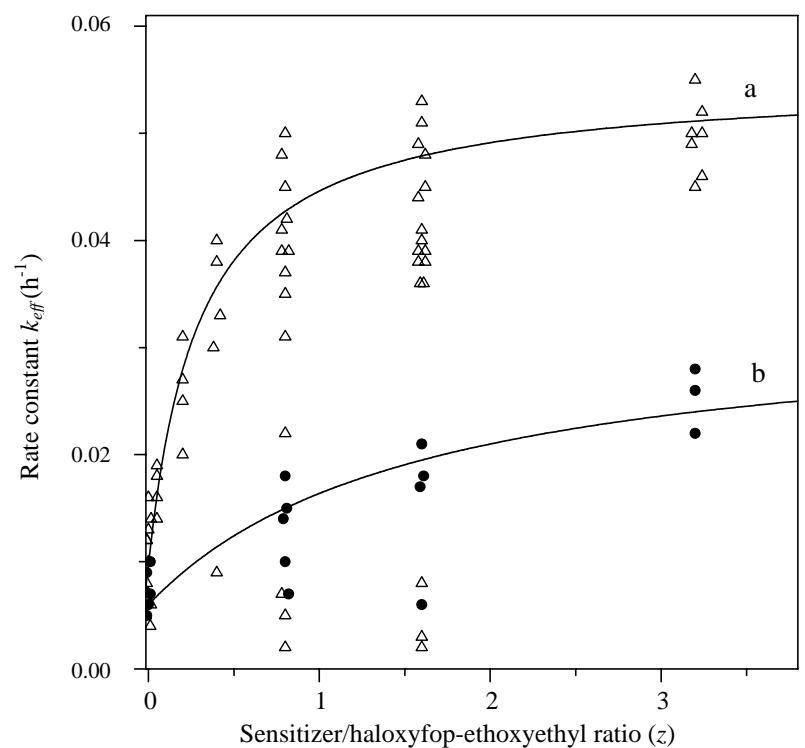

Fig. 4 The decomposition rate constants $k_{\text {eff }}$ in the Zellek-and-sensitizer films as a function of the 'sensitizer/haloxyfop-ethoxyethyl" ratio $z$ : symbols $\Delta$ and the fitted (a)-films $\approx 0.07 \mu \mathrm{m}$ thick irradiated in an oxygen medium (air); • and (b)—in an oxygen-free gas (argon).

\subsection{Mechanisms of Direct and Sensitized Photodecompositions in High Disperse Pesticide Matter}

As follows from the experimental data, no direct photodecomposition of both pesticide chemicals was detected under solar radiation and indoor light (a weak direct decomposition is found in the haloxyfop-ethoxyethyl assays only, Fig. 4). On the other hand, Shirvanol induces photochemical decomposition of the chemicals under study as well as almost all pesticides studied earlier (6 compounds of 8) due to the presence of poly-aromatic ingredients (naphthalene and others), which are the accepted photosensitizers. The physical and chemical reasons for the sensitizing activity of poly-aromatic compounds, and their photochemical fates in the environment are explained in many articles and books [8, 21, 22, 30]. In this regard, the high disperse condition provides an additional chance to induce the photochemical decomposition of pesticide substances in thin films and particles. Actually, after solvent vaporization the residual semi-solid (or viscous) film could consist of a few low-volatile components of the 
initial multi-component formulation. For instance, in the case of sensitized photolysis, the final composition could consist only of a sensitizer compound (the donor of excitation energy) and a pesticide compound (the acceptor). In this composition donor and acceptor are in permanent molecular contact. Such close contact promotes energy transfer from donor to acceptor. It is worth noting that the permanent contact cannot take place in ordinary "test-tube" solutions, in which the solvent serves as a buffer preventing the donor from contact with the acceptor. Moreover, such contacts are unfeasible in thick pesticide particles (films) of tens and hundreds of microns in size. The point is that, taking into account the inverse squared diameter dependence of evaporation rate, one may perceive that the solvent vaporization out of a coarse particle requires many hours and days (certain kinds of solvents used in commercial pesticide formulations are of low and middle volatilities). As a result, the final "donor-and-acceptor" composition hardly forms in thick particulates.

How can the excitation energy transfer from a sensitizer molecule to a pesticide one in the specific case of a high disperse matter? The point is that the conventional singlet-singlet transfer from the photoexcited sensitizer ${ }^{1} \mathrm{D}^{*}$ (donor) to pesticide molecule A (acceptor) is impossible because of the lower excitation level ${ }^{1} \mathrm{D}^{*}$ of sensitizer (which can absorb the solar radiation at $\lambda>300 \mathrm{~nm}$, Fig. 1) as compared with the singlet level ${ }^{1} \mathrm{~A}^{*}$ of pesticide molecule (absorption at $\lambda<300 \mathrm{~nm}$ only). Second, the sensitized decomposition of propiconazole and haloxyfop-ethoxyethyl proceeds with the presence of molecular oxygen, i.e., it is actually the photochemical oxidation induced by sensitizer. The below Eq. (1) to the sensitized photo-oxidative reactions with participation of the intermediate triplet level ${ }^{3} \mathrm{D}^{*}$ and the so-called singlet oxygen ${ }^{1} \mathrm{O}_{2}$ is known (Type II Photooxygenation [8, 30-32]):

$$
\begin{aligned}
& { }^{1} \mathrm{D} \stackrel{h v}{\longrightarrow}{ }^{1} \mathrm{D}^{*} \stackrel{I S C}{\longrightarrow}{ }^{3} \mathrm{D}^{*} \stackrel{{ }^{3} \mathrm{O}_{2}}{\longrightarrow}{ }^{1} \mathrm{O}_{2}{ }^{*} \\
&
\end{aligned}{ }^{1}\left(\mathrm{AO}_{2}\right) \stackrel{h v}{\longrightarrow}\left(\mathrm{AO}_{2}\right)^{*} \rightarrow \text { Products }
$$

where, ${ }^{1} \mathrm{D},{ }^{1} \mathrm{D}^{*}$, and ${ }^{3} \mathrm{D}^{*}$ are the ground state, the excited singlet state, and the excited triplet state of sensitizer, respectively; ${ }^{3} \mathrm{O}_{2}$ is the ground triplet state of oxygen (i.e., ${ }^{3} \mathrm{O}_{2}$ is the molecular oxygen in air), ${ }^{1} \mathrm{O}_{2}$ is the excited singlet oxygen; $\mathrm{A},{ }^{1}\left(\mathrm{AO}_{2}\right)$ and ${ }^{1}\left(\mathrm{AO}_{2}\right)^{*}$ are the pesticide molecule, the intermediate oxidation compound, and its excited state. ISC is the so-called intersystem crossing, i.e., the radiationless transition from the ${ }^{1} \mathrm{D}^{*}$ singlet to triplet ${ }^{3} \mathrm{D}^{*}$ (this process is known to be very efficient in poly-aromatic molecules).

There are several reasons to hypothesize just the oxidative Eq. (1) for photodecomposition of propiconazole and haloxyfop-ethoxyethyl in thin films and particles. First, both propiconazole and haloxyfop-ethoxyethyl decompose with oxygen participation. Second, the poly-aromatic compounds (i.e., the ingredients of Shirvanol) are known to form the triplet levels by ISC easily and, hence, can produce the singlet oxygen ${ }^{1} \mathrm{O}_{2}$. Third, the high disperse condition promotes formation of the (semi-) solid "pesticide-and-sensitizer" particles and films, where triplets ${ }^{3} \mathrm{D}^{*}$ can live long $[7,20,29]$ waiting for a diffusion contact with ${ }^{3} \mathrm{O}_{2}$ (a small quantity of molecular oxygen ${ }^{3} \mathrm{O}_{2}$ dissolves in a pesticide matter) to form the active singlet oxygen ${ }^{1} \mathrm{O}_{2}$. Forth, the propiconazole and haloxyfop-ethoxyethyl molecules have the specific chemical groups (the N-heterocyclic ones) which are known to be suitable for the cycloaddition reactions with singlet oxygen [29, 30]. Fifth, the similar photo-oxidative mechanism was found in thin films and particles made of the earlier studied pesticides resmethrin and fipronil [25, 27]. Based on these arguments, the oxidative reactions may be proposed as follows:

(1) Insertion (cycloaddition) of singlet oxygen into triazole ring of propiconazole (Scheme 3) yielding peroxide I followed by additional absorption of light quantum and decomposition.

(2) Insertion of singlet oxygen into pyridine ring of haloxyfop-ethoxyethyl (Scheme 4) yielding peroxide 


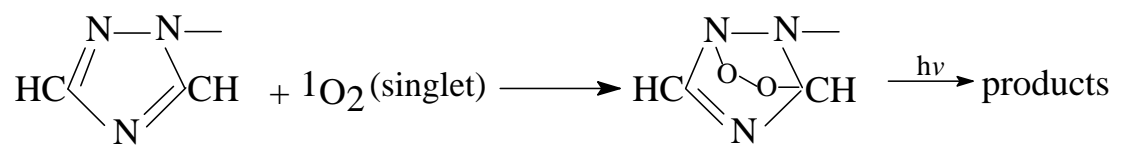

(Propiconazole)
(Peroxide I)

Scheme 3 Insertion (cycloaddition) of singlet oxygen into triazole ring of propiconazole.

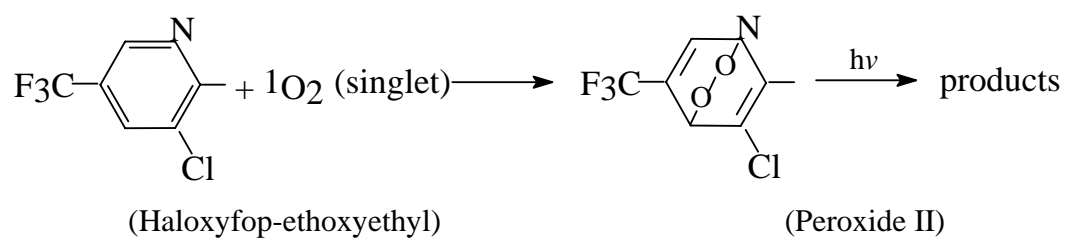

Scheme 4 Insertion of singlet oxygen into pyridine ring of haloxyfop-ethoxyethyl.

II followed by additional absorption of light quantum and decomposition.

A long-time irradiation, 6-12 $\mathrm{h}$ and more, of the propiconazole-and-sensitizer films resulted in $30 \%-50 \%$ decomposition of propiconazole but no chromatographic peaks of products were recorded. However, this is not surprising as such case we observed earlier in experiments with the high disperse particulates [26, 27]. The point is that photolysis induces destruction of a "heavy" pesticide molecule of $300-500 \mathrm{~g} \cdot \mathrm{mol}^{-1}$ yielding several "light" products of 100-150 g.mol ${ }^{-1}$, which should be the volatile compounds. These products evaporate out of thin film just during irradiation and, therefore, the light products cannot be accumulated inside. An attempt was undertaken to record the light products using a sealed narrow quartz tube of $2 \mathrm{~mm}$ ID with the “propiconazole-and-sensitizer' film formed at the inner wall (quartz is transparent for UV). The tube was irradiated during several hours assuming that very small volume of closed tube should prevent full evaporation of light products (this method was earlier successfully applied in Ref. [26]). After the irradiation, the chromatograph recorded four small peaks of light products, but a GC-MS (gas chromatograph-mass spectrometer) failed to recognize them as an amount of each product was too small to be detected (the electron capture detector used in the gas-liquid chromatograph was likely of higher sensitivity). As for the heavy peroxide I, a small peak was observed at chromatogram after the 2-3 $\mathrm{h}$ exposure, which was located at chromatogram behind the propiconazole peak (this fact indicates that this product (peroxide) is "heavier" than propiconazole; in Ref. [27] the similar distant chromatographic peak was recognized with GC-MS as a peroxide). However, after a long-term exposure of 5-10 h this peak collapsed indicating its photochemical instability. Photolysis of the haloxyfop-ethoxyethyl (Zellek) films demonstrated similar behavior, i.e. the absence of light products although the decomposition depth was about $30 \%$. As it was emphasized above, similar phenomenon was observed earlier in the experiments with the high disperse pesticide particulates, including the photochemical instability of intermediate peroxides (e.g., Ref. [25]). It is worth to notice that the common methods (IR, VIS, UV spectroscopy, NMR, optical and electron microscopy) cannot record the chemical and structural changes in a tiny pesticide particle (0.5-1 $\mu \mathrm{m}$ in diameter, 0.1-1 picogram in weight) and thin film under study (1-20 $\mu \mathrm{g}$ in weight, $0.1 \mu \mathrm{m}$ thick), taking into account the permanent evaporation of light products during irradiation.

As follows from Fig. 4, haloxyfop-ethoxyethyl demonstrates a significant oxygen-less sensitized decomposition of $k_{\text {eff }} \approx 0.015-0.025 \mathrm{~h}^{-1}$. This fact indicates that, in addition to the above-stated oxidative Eq. (1), a non-oxidative decomposition takes place in the haloxyfop-ethoxyethyl films. To explain the non-oxidative decomposition, one may assume that 
the primary excitation energy transfers from the ${ }^{1} D^{*}$ singlet to the intermediate ${ }^{3} \mathrm{D}^{*}$ triplet by the intersystem crossing but then the energy can transfer from ${ }^{3} \mathrm{D}^{*}$ to ${ }^{3} \mathrm{~A}^{*}$, i.e., ${ }^{1} \mathrm{D} \stackrel{h v}{\longrightarrow}{ }^{1} \mathrm{D}^{*} \stackrel{I S C}{\longrightarrow}{ }^{3} \mathrm{D}^{*}$ $\stackrel{A}{\longrightarrow}{ }^{3} \mathrm{~A}^{*}$ yeilding the excited triplet ${ }^{3} \mathrm{~A}^{*}$.

Can the excited pesticide molecule ${ }^{3} \mathrm{~A}^{*}$ decay into chemical products? Most likely, the answer is negative as the triplet energy levels lie generally too low to get over the energy barriers of chemical reactions. Nevertheless, the triplet states of both the sensitizer and pesticide molecules can promote the oxygen-less reaction in thin particles and films. The point is that the triplet excitations of the poly-aromatic and $\mathrm{N}$-heterocyclic compounds (i.e., the sensitizer and pesticide molecules) are known to live for a long time in (semi-) solids (i.e., in "sensitizer-and-pesticide" particulates), one second and more at room temperature [8, 21, 33]. Consequently, a significant steady-state concentration of excited triplets ${ }^{3} \mathrm{~A}^{*}$ permanently exists in the irradiating solid matter. It is known that additional absorption of light quantum can occur just from the long-living triplet levels. This absorption results in the double-energy excitation of pesticide molecules $\mathrm{A}^{* *}$ followed by chemical decay (the "two-quantum photochemistry" [33]):

$$
\begin{aligned}
& { }^{1} \mathrm{D} \stackrel{h v}{\longrightarrow}{ }^{1} \mathrm{D}^{*} \stackrel{\mathrm{ISC}}{\longrightarrow}{ }^{3} \mathrm{D}^{*} \stackrel{A}{\longrightarrow}{ }^{3} \mathrm{~A}^{*} \stackrel{h v}{\longrightarrow} \\
& \rightarrow{ }^{3} \mathrm{~A}^{* *} \rightarrow \text { Products }
\end{aligned}
$$

Besides, a mechanism combining the two-quantum excitation and formation of the ground complexes (D...A) (Scheme 3 below) or/and the excited complexes (D...A ${ }^{*}$ (Scheme 4) can be proposed for the non-oxidative sensitized decomposition [31]:

$$
\begin{gathered}
\mathrm{D}+\mathrm{A} \rightarrow(\mathrm{D} \ldots \mathrm{A}) \stackrel{h v}{\longrightarrow}{ }^{1}(\mathrm{D} \ldots \mathrm{A})^{*} \stackrel{I S C}{\longrightarrow}{ }^{3}(\mathrm{D} \ldots \\
\mathrm{A})^{*} \stackrel{h v}{\longrightarrow}{ }^{3}(\mathrm{D} \ldots \mathrm{A})^{* *} \rightarrow \mathrm{D}+\text { Products } \\
\mathrm{D} \underset{h v}{\stackrel{h v}{\longrightarrow}}{ }^{3} \mathrm{D}^{*} \stackrel{I S C}{\longrightarrow}(\mathrm{D} \ldots \mathrm{A})^{* *} \rightarrow \mathrm{D}+\text { Products } \\
\stackrel{3}{\longrightarrow}(\mathrm{D} \ldots \mathrm{A})^{*}
\end{gathered}
$$

Evidently, formation of the intermediate (D...A) and ${ }^{3}(\mathrm{D} \text {...A })^{*}$ complexes should be very efficient if molecules $\mathrm{D}$ and $\mathrm{A}$ are permanently in close molecular contact. The intersystem crossing ISC is known to be very efficient in poly-aromatic molecules.
The two-quantum photochemistry proceeds effectively in the solid poly-aromatic and N-heterocyclic matter, where neither translational motion of molecules nor free rotation of molecular segments takes place [33]. As it was explained in Section 1.1, such conditions arise in the (semi-) solid particles and films formed by liquid solvents evaporation out of a pesticide-and-sensitizer mixture. Consequently, it is valid to assume that the high disperse conditions in thin films and particles should be favorable for the sensitizer-and-pesticide complexing followed by ISC and the two-quantum excitation (i.e., for Eqs. (3) and (4)).

An experimental evidence can be advanced in favor of the above complexing mechanism. Fig. 5 shows the absorption spectrums of pesticides acrinathrin and propiconazole, and of sensitizer Shirvanol, recorded as both the individual substances and their composites. The spectrums were recorded in both thin (semi-) solid films on quartz plates and in liquid solutions. The partial concentrations were the same in the individual films (solutions) and the composite ones (as the thickness of optical cell with liquid solution is $1 \mathrm{~cm}$, the volume concentration in solution, $\mathrm{C} \mu \mathrm{g} \cdot \mathrm{mL}^{-1}$, is numerically equal to the surface concentration in film, C $\mu \mathrm{g} \cdot \mathrm{cm}^{-2}$ ). As seen in Fig. 5, the absorption of the "acrinathrin-and-Shirvanol" composite film is not merely the sum of absorptions of the individual acrinathrin and Shirvanol films. In particular, the composite film absorbs much more light at $\lambda>300$ $\mathrm{nm}$, where acrinathrin should contribute nothing to the overall absorption. Besides, two local peaks, $\approx$ 320-330 $\mathrm{nm}$ and $\approx 335-345 \mathrm{~nm}$, are more apparent and shifted in the composite film, but they are weaker in the sensitizer film. On the contrary, the liquid "acrinathrin-and-Shirvanol" solution shows the ordinary spectral sum of individual solutions of acrinathrin and Shirvanol (a minor difference between the mix spectrum and the individual spectrums is very likely due to the inaccuracies in the concentrations prepared). The "propiconazole-and-Shirvanol” composite film, and the individual propiconazole and 


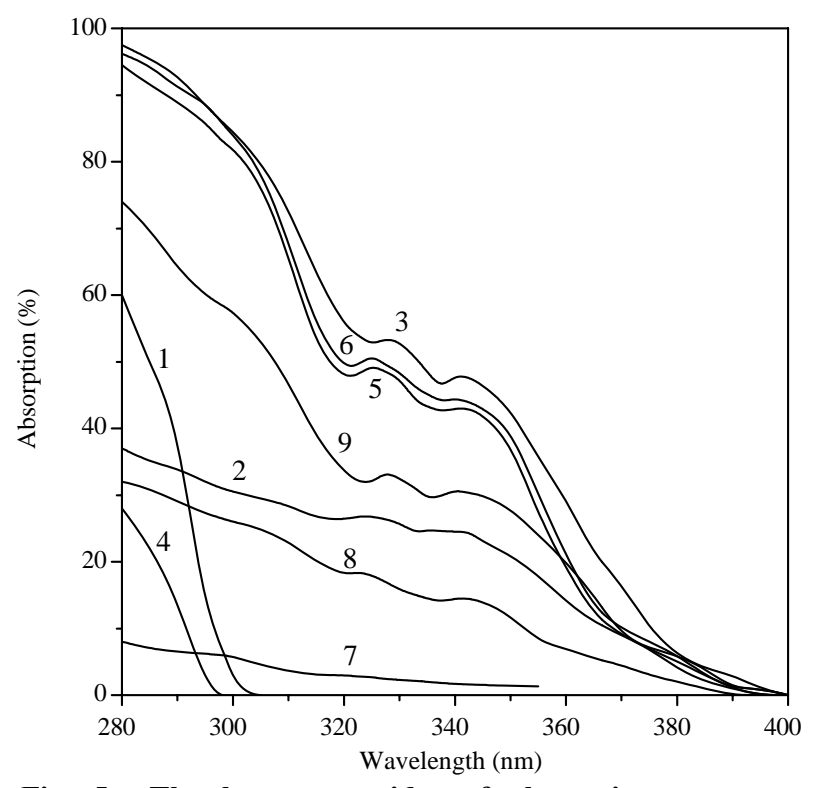

Fig. 5 The long-wave sides of absorption spectrums: 1-3-acrinathrin film $25 \mu \mathrm{g} \cdot \mathrm{cm}^{-2}$; Shirvanol film $20 \mu \mathrm{g} \cdot \mathrm{cm}^{-2}$; and composite film "acrinathrin $25 \mu \mathrm{g} \cdot \mathrm{cm}^{-2}+$ Shirvanol 20 $\mu \mathrm{g} \cdot \mathrm{cm}^{-2 \%}$, respectively; 4-6-acrinathrin solution (isopropanol) $32 \mu \mathrm{g} \cdot \mathrm{mL}^{-1}$, optical cell $1 \mathrm{~cm}$ thick; Shirvanol solution $20 \mu \mathrm{g} \cdot \mathrm{mL}^{-1}$; and composite solution "acrinathrin 32 $\mu \mathrm{g} \cdot \mathrm{mL}^{-1}+$ Shirvanol $20 \mu \mathrm{g} \cdot \mathrm{mL}^{-1}$ ", $\quad$ respectively; 7-9-propiconazole film $25 \mu \mathrm{g} \cdot \mathrm{cm}^{-2}$; Shirvanol film 16 $\mu \mathrm{g} \cdot \mathrm{cm}^{-2}$; and composite film "propiconazole $25 \mu \mathrm{g} \cdot \mathrm{cm}^{-2}+$ Shirvanol $16 \mu \mathrm{g} \cdot \mathrm{cm}^{-2}$, , respectively.

Shirvanol films demonstrate similar behavior. It may be then assumed that the complexes (D...A) actually arise in thin (semi-) solid composite pesticide films but not in liquid solutions. There is no practical way to discriminate separately the complexing Eqs. (3) and (4), and the two-quantum Eq. (2) as Eqs. (3) and (4) include both the complex formation and the two-quantum absorption, i.e. both reactions proceed cooperatively.

\section{Conclusions}

Photochemical decompositions of two commercial pesticide formulations, Tilt (the active ingredient propiconazole) and Zellek (haloxyfop-ethoxyethyl), were studied in the form of thin films $\approx 0.04-0.2 \mu \mathrm{m}$ thick, and fine particles of $\approx 0.12-1.3 \mu \mathrm{m}$ in diameter. Study of the reactions in the particulates and films made of the commercial pesticide formulations is caused by the specificity of physicochemical conversions and chemical reactions in the chemically multi-component substances. The chemical and phase conditions, and the bulk structures of final particles and films form in the course of the gradual volatile solvents evaporation out of particles (films) followed by solidification and stratification of the remaining matter. Therefore, the chemical, phase, dispersal and bulk properties of pesticide particles and films depend on chemical and physicochemical conditions of both pesticide ingredients and non-pesticide constituents of the initial formulations. The expressivity of these conditions and properties is diverse, spontaneous and often irreproducible. Spontaneity and irreproducibility of chemical and structural conditions are the specific feature of (photo-) chemical reactions in high disperse particulates.

As it can be estimated from data on the reaction rates, the haloxyfop-ethoxyethyl (Zellek) residues formed on foliage upon pesticide treatments of agricultural fields would essentially decompose in 4-6 weeks via the direct photoreactions under sunlight $\left(k_{\text {eff }}\right.$ $\approx 0.007-0.01 \mathrm{~h}^{-1}$ ). Direct decomposition of the propiconazole (Tilt) contaminants would possibly need more time because of lack of absorption of the near-land UV light. The sensitized oxidative and non-oxidative decompositions of pesticide residues on foliage would also possibly proceed as the plant leaves contain the native (poly-) aromatic and porphyrin compounds, which could act as the native sensitizers (e.g., chlorophyll is an excellent photosensitizer for all types of photooxidation [31]).

\section{References}

[1] Atkinson, R.; Guicherit, R.; Hites, R. A.; Palm, W. U.; Seiber, J. N.; Voogt, P. Transformations of Pesticides in the Atmosphere-a State-of-the-art. Water, Air and Soil Pollution 1999, 115, 195-218.

[2] Atkinson, R. Gas-phase Tropospheric Chemistry of Organic Compounds: A Review. Atmos. Environ. 2007, 41, 200-240.

[3] Seinfeld, J. H.; Pandis, S. N. Atmospheric Chemistry and Physics: From Air Pollution to Climate Change; Wiley: Hoboken, 2006; p 1203.

[4] Calvert, J. G.; Atkinson, R.; Becker, K. H.; Kamens, R. 
M.; Seinfeld, J. H.; Wallington, T. J.; et al. The Mechanisms of Atmospheric Oxidation of the Aromatic Hydrocarbons; Oxford Press: NY, 2002; p 556.

[5] Rudich, Y. Laboratory Perspectives on the Chemical Transformation of Organic Matter in Atmospheric Particles. Chem. Rev. 2003, 103, 5097-5124.

[6] Kanakidou, M.; Seinfeld, J. H.; Pandis, S. N.; Barnes, I.; Dentener, F. J.; Facchini, M. C.; et al. Organic Aerosols and Global Climate Modelling: A Review. Atmos. Chem. Phys. 2005, 5, 1053-1123.

[7] Boule, P.; Bachemann, D. W. Handbook of Environmental Chemistry: Environmental Photochemistry; Springer: Berlin Heidelberg, 1999; p 359.

[8] Boule, P.; Bachemann, D. W.; Robertson, P. K. J. Handbook of Environmental Chemistry: Environmental Photochemistry; Springer: Berlin Heidelberg, 2005; p 489.

[9] Gross, S.; Bertram, A. K. Reactive Uptake of $\mathrm{NO}_{3}, \mathrm{~N}_{2} \mathrm{O}_{5}$, $\mathrm{NO}_{2}, \mathrm{HNO}_{3}$ and $\mathrm{O}_{3}$ on Three Types of Polycyclic Aromatic Hydrocarbon Surface. J. Phys. Chem. A 2008, 112, 3104-3113.

[10] Segal-Rosenheimer, M.; Linker, R.; Dubowski, Y. Heterogeneous Oxidation of the Insecticide Cypermethrin as Thin Film and Airborne Particles by Hydroxyl Radicals and Ozone. Phys. Chem. Chem. Phys. 2010, 13, 506-517.

[11] Wang, Y.; Zhang, P.; Yang, B.; Liu, C.; Shu, J. Kinetic and Product Study of Heterogeneous Reactions of $\mathrm{NO}_{3}$ Radicals with Suspended Resmethrin, Phenothrin and Fenvalerate Particles. Chemosphere 2013, 90, 848-855.

[12] Eliason, T. L.; Aloisio, S.; Donaldson, D. J.; Cziczo, D. J.; Vaida, V. Processing of Unsaturated Organic Acid Films and Aerosols by Ozone. Atmos. Environ. 2003, 37, 2207-2219.

[13] Eliason, T. L.; Gilman, J. B.; Vaida, V. Oxidation of Organic Films Relevant to Atmospheric Aerosols. Atmos. Environ. 2004, 38, 1367-1378.

[14] Ueda, K.; Gaughan, L. C.; Casida, J. E. Photodecomposition of Resmethrin and Related Pyrethroids. J. Agric. Food Chem. 1974, 22, 212-220.

[15] Ivie, G. W.; Casida, J. E. Sensitized Photodecomposition and Photosensitizer Activity of Pesticide Chemicals Exposed to Sunlight on Silica Gel Chromatoplates. J. Agric. Food Chem. 1971, 19, 405-409.

[16] Holmstead, R. L.; Casida, J. E.; Ruzo, L. O.; Fullmer, D. G. Pyrethroid Photodecomposition: Permethrin. J. Agric.
Food Chem. 1978, 26, 590-595.

[17] Ruzo, L. O.; Smith, I. H.; Casida, J. E. Pyrethroid Photochemistry: Photooxidation Reactions of the Chrysanthemates Phenothrin and Tetramethrin. J. Agric. Food Chem. 1982, 30, 110-115.

[18] Ruzo, L. O.; Casida, J. E.; Holden, I. Pyrethroid Chemistry: Reactive $\alpha, \beta$-Unsaturated Keto Aldehydes from Peracid Oxidation, Oxidative Photodecomposition, and Metabolism of 5-benzyl-3-furylmethyl Derivatives. $J$. Agric. Food Chem. 1985, 33, 622-625.

[19] Segal-Rosenheimer, M.; Dubowski, Y. Photolysis of Thin Films of Cypermethrin using the FTIR Monitoring: Products, Rates and Quantum Yields. J. Photochem. Photobio. A 2008, 200, 262-269.

[20] Randy, B.; Rabek, J. F. Photodegradation, Photo-Oxidation and Photostabilization of Polymers; Wiley: NY, 1975; p 573.

[21] Rabek, J. F. Photodegradation of Polymers: Physical Characteristics and Applications; Springer: Berlin-Heidelberg, 1996; p 212.

[22] Shlyapintokh, V. J. Photochemical Transformations and Stabilization of Polymers; Khimija: Moscow, 1979; p 344.

[23] Samsonov, Y. N., Makarov, V. I.; Koutsenogii, K. P. Physicochemical Model and Kinetics of Pesticide Constituent Evaporation Out of Multi-ingredient Polydisperse Aerosols. Pest. Sci. 1998, 52, 292-302.

[24] Ivie, G. W.; Casida, J. E. Photosensitizers for the Accelerated Degradation of Chlorinated Cyclodienes and Other Insectice Chemicals Exposed to Sunlight on Bean Leaves. J. Agric. Food Chem. 1971, 19, 410-416.

[25] Samsonov, Y. N.; Makarov, V. I. Kinetics and Photophysical Mechanism of Sunlight Photolysis of Unstable Resmethrin and Phenothrin in Aerosols and Thin Films. Bull. Environ. Contam. Toxic. 1996, 56, 903-910.

[26] Samsonov, Y. N.; Pokrovskii, L. M.; Sensitized Photodecomposition of High Disperse Pesticide Chemical Exposed to Sunlight and Irradiation from Halogen or Mercury Lamp. Atmos. Environ. 2001, 35, 2133-2141.

[27] Samsonov, Y. N. Physicochemical Transformation and Photochemical Reactions in High-Disperse Substance State: Chemical Decomposition in Aerosols and Thin Films Made from Pesticide Fipronil (Adonis). J. Atmos. Chem. 2007, 56, 127-147.

[28] Gasanov, K. G.; Mamedov, A. P.; Salmanova, C. K.; Djaforova, R. A.; Suleimenova, M. K.; Mamedov, A. K. Method of Preparing of Petroleum Luminophore 

Relevant to Environmental Conditions

Concentrate. Bulletin of Inventions 1995, 6.

[29] Willeke, K.; Baron, P. Aerosol Measurement: Principles, Techniques and Application; Wiley: NY-Toronto, 1993; p 896.

[30] Gollnick, K. Type II Photooxygenation Reactions in Solutions. In Advances in Photochemistry: V6; Noyes, W. A., Hammond, G. S.. Pitts, J. N. J., Eds.; Wiley:
NY-Toronto, 1968; pp 1-122.

[31] Foote, C. S. Mechanism of Photosensitized Oxidation. Science 1968, 3857, 963-970.

[32] Rappoport, Z. The Chemistry of Functional Groups: The Chemistry of Peroxides; Wiley: Chichester, 2006; p 1524.

[33] Bagdasaryan, K. S. Two-Quantum Photochemistry. Nauka: Moscow, 1976; p 128. 